\title{
Feasibility study of a time-resolving x-ray tomographic system
}

\author{
R F Mudde ${ }^{1}$, J Alles ${ }^{1}$ and T H J J van der Hagen ${ }^{2}$ \\ ${ }^{1}$ Kramers Laboratorium voor Fysische Technologie, Delft University of Technology, Pr. Bernhardlaan 6, \\ 2628 BW Delft, The Netherlands \\ ${ }^{2}$ Reactor Physics Department, Delft University of Technology, Mekelweg 15, 2629 JB Delft, \\ The Netherlands \\ E-mail: r.f.mudde@tudelft.nl
}

Received 1 November 2007, in final form 29 May 2008

Published 30 June 2008

Online at stacks.iop.org/MST/19/085501

\begin{abstract}
This paper discusses the possibilities for developing a tomographic scanner for studying the phase distribution of fluidized beds. The system is based on a medical x-ray source equipped with $30 \mathrm{CdWO}_{4}$ detectors. We mimic a five-source system via simulation and in experiments of voids in a 23 fluidized bed filled with polystyrene particles. Both static voids and moving ones are studied. The reconstruction uses the simultaneous algebraic reconstruction technique with regularization. We find that it is possible to reconstruct objects with a spatial resolution of about $5 \mathrm{~mm}$ at a frame rate of $200 \mathrm{~Hz}$. It is concluded that noise levels should be kept below $2 \%$.
\end{abstract}

Keywords: $\mathrm{x}$-ray tomography, time-resolved, SART, regularization, fluidized bed

\section{Introduction}

Fluidized beds are frequently encountered in many industrial applications. They consist of a cylinder containing a large quantity of small particles through which gas is flowing upwards. The gas velocity is such that the particles 'float' on the gas stream, creating a gas-particle mixture that has fluid-like properties. Our understanding of fluidization is still far from complete, although during the last decade computational methods have increased our knowledge significantly. However, due to their opaqueness, fluidized beds create serious problems for experimentalists. The well-known laser-based techniques developed for single phase flows are inadequate for fluidized systems. Consequently, experiments on local phenomena are rare. However, solids are to some extent transparent to radiation such as $\mathrm{x}$ - or $\gamma$-rays and a variety of nuclear techniques have been developed during the last decade. In Duduković (2000) the use of nuclear techniques in opaque multiphase systems is reviewed.

Radiation can be used to measure the volume fraction of gas (or equivalent of the solids) in a fluidized bed. If tomographic techniques are used, the volume fraction distribution in a cross section of the fluidized bed can be found. This is referred to as 'nuclear densitometry'. Several papers have appeared in the literature, not only dealing with densitometry in fluidized beds, see e.g. Kumar et al (1995), Kumar et al (1997) and Kumar and Duduković (1997). For instance, Shollenberger et al (1997) investigated the volume fraction in a $48 \mathrm{~cm}$ diameter bubble column, using a single beam-detector pair to perform horizontal, unidirectional scans over a cross-sectional plane. Provided cylinder symmetry holds, the measurements contain enough information to reconstruct the time-averaged volume fraction of the gas phase. Mudde et al (1999) followed a similar procedure to measure the solid volume fraction in a turbulent fluidized bed. Kumar et al (1995) reported the use of a fan beam from a single $\gamma$ source with multiple detectors. They rotated the sourcedetector combination around the column taking about 4000 projections. This way they could reach a spatial resolution of $5 \mathrm{~mm}$. Since the total measurement time is of the order of $1 \mathrm{~h}$, only a time-averaged volume fraction distribution is reconstructed. This type of tomographic reconstruction is referred to as computed tomography or CT. In medical imaging, the use of x-ray tomography is standard practice. The source-detector system again rotates around the patient. This way high-resolution, static images can be produced.

The nuclear techniques compete with techniques based on electromagnetic fields, like electrical impedance tomography. 
These EM methods are generally much faster, giving a high temporal resolution. Frame rates of several hundreds per second can easily be achieved (see, e.g., Reinecke and Mewes (1996), Kühn et al (1996), Beck et al (1998)). However, the EM techniques suffer from soft-field effects characteristic of electrical methods, i.e. a change in the electromagnetic properties in one location changes the field everywhere in the domain. This causes poor resolution in the center of the object. Nuclear densitometry relies on hard fields. Hence, they do not suffer from this problem. But, the nuclear techniques are rather slow. Due to inherent noise, the frame rate (temporal resolution) that can be obtained is usually low. High spatial resolution requires relatively long measuring times as compared to the impedance techniques, slowing down this technique.

An ultra fast $\mathrm{x}$-ray tomographic system has been developed by Hampel and co-workers, see e.g. Bieberle and Hampel (2006) and Bieberle et al (2007). They generate the $\mathrm{x}$-rays by scanning a tungsten element with a fast moving electron beam. This way, it is possible to create tomographic images up to a rate of 10000 frames $\mathrm{s}^{-1}$. This is evidently showing that nuclear techniques can also be fast.

In the present paper, we focus on the use of $\mathrm{x}$-rays for tomographic imaging. The application we have in mind is a bubbling fluidized bed. We aim at time-resolved measurements, hence the actual sampling time per image needs to be small. We have as our target to be able to generate more than 100 images s $^{-1}$ (see Mudde et al (2005)).

In this paper, we present simulations and experiments on voids in a fluidized bed, using a standard medical $\mathrm{x}$-ray source operating in the fan-beam mode, with 30 detectors. The voids are measured from five different angles, mimicking a 5-source system. The measuring time per angle, however, is kept short, i.e. of the order of $10 \mathrm{~ms}$. The data are reconstructed with an extension of the so-called SART algorithm by invoking regularization based on a median filter. In section 2 , the experimental setup is discussed. Section 3 describes the reconstruction algorithm used. Computer simulations are presented in section 4. Reconstructions of measured data are given in section 5. We discuss the influence of various experimental settings on the data and will show that a frame rate of 400 frames $\mathrm{s}^{-1}$ is feasible. Finally, we present reconstruction of voids moving through the measuring plane at a velocity up to $60 \mathrm{~cm} \mathrm{~s}^{-1}$.

\section{Experimental setup}

The fluidized bed is formed by a $23 \mathrm{~cm}$ inner-diameter tube (perspex, wall thickness $5 \mathrm{~mm}$ ). The particles are $2 \mathrm{~mm}$ sized polystyrene particles. This polystyrene has a density of $1.06 \times 10^{3} \mathrm{~kg} \mathrm{~m}^{-3}$. The mean density of loosely packed polystyrene particles is $625 \mathrm{~kg} \mathrm{~m}^{-3}$. In the bubbling regime of fluidization, voids travel upwards through the powder mass. A schematic of a fluidized bed with two bubbles in the measuring plane of the densitometer is given in figure 1 .

In our experiments, the voids are mimicked by placing empty cylinders (perspex, wall thickness $1.5 \mathrm{~mm}$ ) inside the particle phase. The fluidized bed is positioned on a rotating

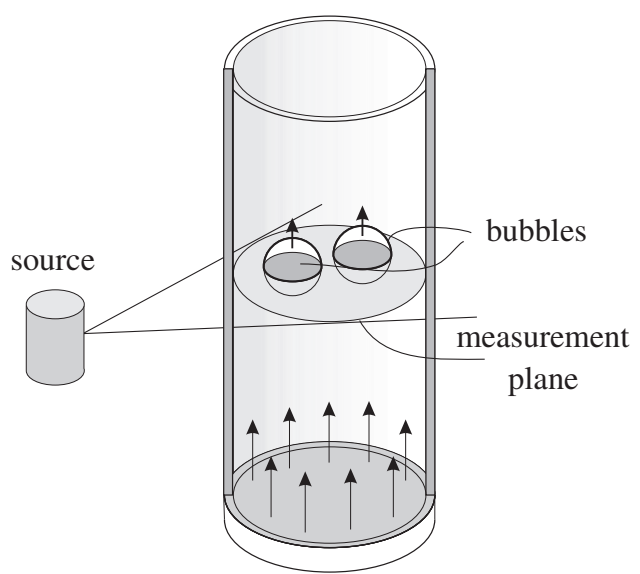

Figure 1. Fluidized bed with two bubbles in the measuring plane of the $\mathrm{x}$-ray densitometer.

Table 1. X-ray tube data.

\begin{tabular}{ll}
\hline $\begin{array}{l}\text { X-ray tube } \\
\text { Manufacturer }\end{array}$ & Philips \\
Type & SRO 25 50-ROT 350 \\
Voltage (peak) & $150 \mathrm{kV}$ \\
$\begin{array}{l}\text { Anode material } \\
\text { Anode angle }\end{array}$ & $\begin{array}{l}\text { Rhenium alloyed tungsten molybdenum } \\
15^{\circ}\end{array}$ \\
$\begin{array}{l}\text { Anode diameter } \\
\text { Inherent filtering }\end{array}$ & $90 \mathrm{~mm}$ \\
$\begin{array}{l}\text { Tube collimator } \\
\text { Type }\end{array}$ & $980460261501 \mathrm{mD} \mathrm{Al} / 90 \mathrm{kV}$ \\
$\begin{array}{l}\text { X-ray generator } \\
\text { Type }\end{array}$ & Super 100 CP \\
Ripple & 12 -pulse equivalent \\
\hline
\end{tabular}

table, with its axis coinciding with the rotation axis of the table. The x-ray source is placed on one side of the fluidized bed and the array of sensors on the other. The scanner setup is schematically shown in figure 2 . The distance from the x-ray target to the center of the fluidized bed is $68.5 \mathrm{~cm}$ and from the $\mathrm{X}$-ray target to the detectors is $138.6 \mathrm{~cm}$.

The x-ray source used is of standard medical type. All relevant data are summarized in table 1.

The x-ray tube is equipped with a depth collimator. The collimator has a hand-operated diaphragm with which the $\mathrm{x}$ ray beam size can be adjusted. To visualize the selected beam size, the collimator is equipped with a halogen lamp that produces a light beam that coincides with the $\mathrm{x}$-ray beam. The $\mathrm{x}$-ray tube is in principle fully operated via the control panel that has been customized to allow triggered operation.

The detectors of the tomographic scanner all consist of a $\mathrm{CdWO}_{4}$ scintillation crystal optically coupled to a PIN photo diode. Details are given in table 2.

The $\mathrm{x}$-ray fan beam diverges slightly in the vertical direction, with an angle of less than $1^{\circ}$. This causes the beam height to be more than the crystal diameter. Therefore, the beam is collimated at the detector side. In the design of the current scanner setup it has been chosen not to collimate each detector individually. Instead a collective collimator has been built, consisting of a $5 \mathrm{~mm}$ thick lead shield with a narrow slit, as can be seen in figure 2. The width of the slit is $7 \mathrm{~mm}$. 

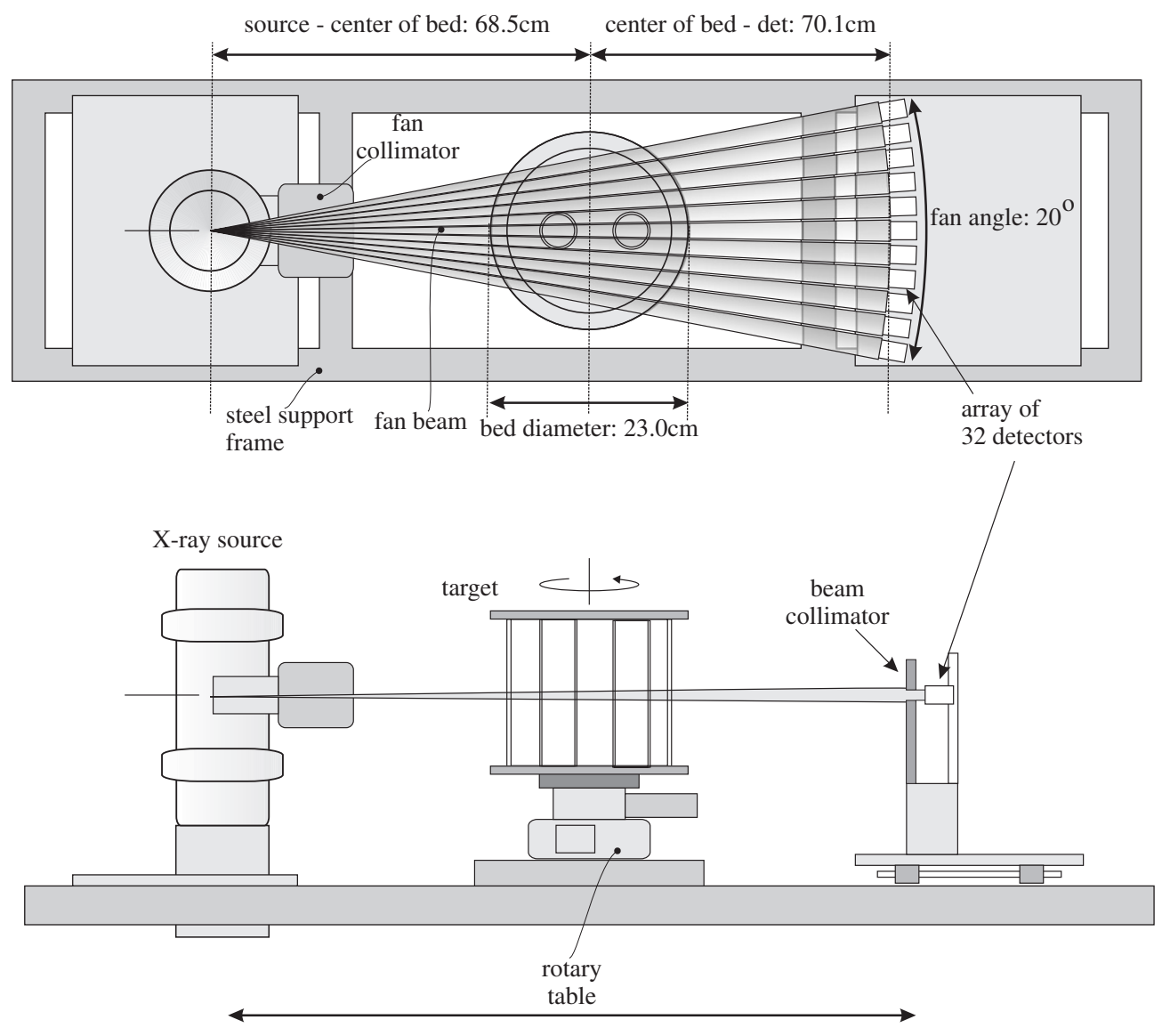

source-detector distance $=138.6 \mathrm{~cm}$

Figure 2. Schematic plan and side views of the tomographic x-ray scanner.

Table 2. Scintillator crystal-photo diode assembly.

\begin{tabular}{ll}
\hline Scintillation crystal & \\
Type & $\mathrm{V} 10 \mathrm{C} 10 \mathrm{CdWO}_{4}$ \\
Dimensions & $10 \mathrm{~mm} \times 10 \mathrm{~mm} \times 10 \mathrm{~mm}$ \\
Housing & Aluminum \\
Decay time & $14 \mu \mathrm{s}$ \\
Emission maximum & $475 \mathrm{~nm}$ \\
Scintillation yield & $12-15$ (photons $\left.\mathrm{keV}^{-1}\right)$ \\
Photo diode & \\
Manufacturer & Hamamatsu \\
Type & $\mathrm{S} 1337-1010 \mathrm{BR}$ \\
\hline
\end{tabular}

As a consequence, the thickness of the x-ray sheet through the bed that is seen by the detectors is approximately $5 \mathrm{~mm}$. The scintillation crystal of each detector is encapsulated in a cylindrical aluminum housing with a diameter of $15 \mathrm{~mm}$, causing a detector-detector distance of $16 \mathrm{~mm}$.

A curved plastic casing holds a horizontal array of 32 detectors. The curvature of the array is such that the distance to the focal point of the source is equal for all detectors (see top view in figure 2). Of these detectors 30 are used to produce data for tomographic reconstruction, the two outermost detectors are used to monitor the alignment of the object vessel. Obviously, the rays that travel to the outer detectors of the array encounter less material (and hence less
Table 3. Specifications of the A/D converter boards.

\begin{tabular}{ll}
\hline$A / D$ converter board & \\
Manufacturer & Spectrum \\
Type & MI.31xx \\
Number of channels & 8 \\
Data width & $12 \mathrm{bit}$ \\
Maximum sample rate & $6.25 \mathrm{MS} \mathrm{s}^{-1}$ \\
\hline
\end{tabular}

attenuation) on their paths, compared to rays traveling to the central detectors. Additional copper filtering has been applied to the outer ray paths, such that irradiation of the filled vessel yields detector signals that are more or less within the same range.

Detector data are acquired by four $2 \mathrm{GHz}$ PCs, equipped with eight channel A/D converter boards (see table 3 ).

Obviously, the maximum attainable speed of the A/D boards is far beyond the required one. In different experiments, we used BGO crystals that allowed measurement of single photons. This requires high speed. Here the data acquisition speed per detector is $25 \mathrm{kHz}$. The amplified analogue output signal of the photo diodes is converted to 12 bits binary data. The A/D converter boards can be operated in trigger mode. In this mode, the boards will start collecting a predefined amount of data at a set sample rate, when they receive an external trigger signal. 


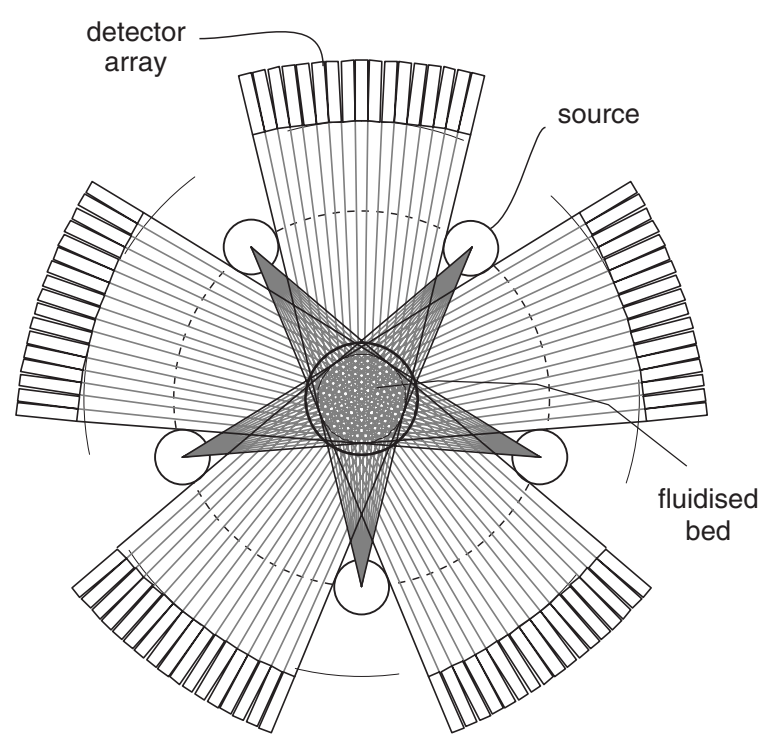

Figure 3. Five-source fan-beam configurations.

\section{Limited data $x$-ray tomography}

\subsection{Configuration}

There are a number of steps in the process from measurement to tomogram, that determine the accuracy. One of them is the noise on the measured data, while a second one is the choice of the reconstruction algorithm. Previous work has shown (see Mudde et al (2005)) that we need a five-source system as illustrated in figure 3. From that study, it was clear that using three sources was insufficient to get the required accuracy. Behind reconstructed objects, as seen from the source, a noisy tail appeared that corrupted the interpretation.

\subsection{Measuring principle and reconstruction algorithm}

When a narrow, parallel beam of mono-energetic $\gamma$ - or x-rays is transmitted through a closed system containing a particlegas two-phase mixture, the number of photons registered per second, $R$, follows from the Lambert-Beer law and can be written as

$$
R=R_{0} \exp \left[-\left((1-\alpha) \mu_{p}+\alpha \mu_{g}\right) d\right]
$$

where $R_{0}$ is the number of photons registered per second when the system is empty, $\mu_{p}$ and $\mu_{g}$ denote the linear absorption coefficient of the particle and gas phases, $\alpha$ is the volume fraction of the gas phase and $d$ is the inner diameter of the system. It should be noted that the attenuation characteristics of the fluidized bed wall is incorporated in $R_{0}$. Further note that both $\mu_{p}$ and $\mu_{g}$ are in principle functions of the photon energy $E$. An x-ray source generates a wide spectrum of photon energies. It can be shown (see, e.g., Alles and Mudde (2007)) that the attenuation of the photons beam can be described with reasonable accuracy by using an effective energy $E_{\text {eff }}$ : the attenuation of the photon beam is then described via the corresponding $\mu\left(E_{\text {eff }}\right)$. However, instead of using an effective energy, we calibrate the detector response as a function of the fraction of solids along the $\mathrm{x}$-ray beams. The calibration curve is accurately described by $A_{\text {cal }}+B_{\text {cal }} \exp \left(-x / C_{\text {cal }}\right)$, with $x$ being the distance traveled by the beam through the powder phase, see figure 9.

Using this calibration, the data measured in an experiment can be converted to a measured distance, $x_{\text {meas }}$, the beam travels through the powder mass. By using $N_{s}$ different sources, with $N_{r}$ measured beams per source, we have $N_{s} \cdot N_{r}$ independent measurements through the object.

Tomographic reconstruction. Different strategies are used for subtracting the required information from the measured data. If sufficient a priori information on the objects to be reconstructed is available, a parametric reconstruction can be used. In this approach a number of objects at unknown positions and of unknown size, but with known shape, are searched for (see West et al (2000) and Bissessur and Peyton (2005)). In the present case we used the general methods, that do not use such a priori information. These reconstruction methods can be broadly classified into two groups: analytic and algebraic. The analytic methods are based on the deconvolved backprojection, e.g. using the Fourier transform and while accurate and fast, they require uniform and closely spaced attenuation data. The filtered back projection algorithm is a popular member of this family. In contrast, algebraic techniques are iterative and reconstruct the object function on a discretized domain. Although significantly slower, algebraic methods offer more flexibility in terms of limited data sets and are more appropriate for the CT configuration system under consideration here. Detailed accounts of reconstruction techniques can be found in Brooks and DiChiro (1976), Herman (1980) or Kak and Slaney (1988).

The iterative methods are based on a finite series expansion (see Herman and Lent (1976)). It is assumed that a finite series of basis functions exists, whose linear combination can provide an adequate approximation to the spatial distribution of the measured quantity. In the present case, we use the calibration procedure to convert the measured line-averaged attenuation into a line-averaged solids fraction. This means that the solids fraction $\alpha(x, y)$ is the quantity to be reconstructed. For the basis functions an easy option is to choose pixels. This choice has several advantages: (i) simplicity, (ii) standard way of discretizing a digital picture, (iii) it leads to a maximally sparse system matrix, which speeds up the reconstruction. The unknown field $\alpha(x, y)$ is thus estimated as

$$
\tilde{\alpha}(x, y)=\sum_{k=1}^{N} \tilde{\alpha_{k}} \phi_{k} .
$$

The basis functions, $\left\{\phi_{k}\right\}$, can be omitted by adopting a lexicographic ordering for the pixels. For a given ray, traveling through the object, the total solids fraction on the line, $p_{i}$, referred to as ray sum, can be estimated as

$$
\tilde{p}_{i}=\sum_{k=1}^{N} W_{i k} \alpha_{k}
$$

with $\alpha_{k}$ being the pixel-based value of the solids fraction distribution and $W_{i k}$ the weighing factor for pixel $k$ for the $i$ th ray through the object. We use a linear weighing matrix 


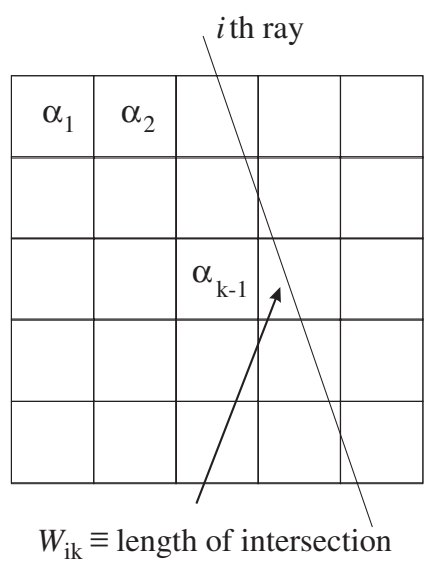

Figure 4. Coordinate system.

$W$. Hence, the weighing factor $W_{i k}$ is the length of ray $i$ through pixel $k$ (see figure 4).

The image reconstruction problem can now be formulated as solving the unknown pixel-averaged solids fraction $\alpha_{k}$ from equation (3) for $M$ different rays and $N$ pixels.

In general, the above problem is ill-posed in the sense of Hadamard. This is a.o. due to noise in the measured ray sums and the approximate nature of the finite series expansion. We write more formally

$$
\vec{p}=\mathbf{W} \cdot \vec{\alpha}+\vec{\epsilon}
$$

where $\vec{\epsilon}$ contains all errors.

The algebraic reconstruction techniques (see, e.g., Brooks and DiChiro (1976)) are concerned with minimizing the mismatch between the data $\vec{p}$ and $\mathbf{W} \cdot \vec{\alpha}$. They do not consider the stochastic errors contained in $\epsilon$. For this reason, they are referred to as deterministic models.

The ART method, introduced by Gordon et al (1970), is an iterative method that solves $\vec{p}=\mathbf{W} \cdot \vec{\alpha}$. A number of refinements on the ART method have been proposed. We have used the simultaneous algebraic reconstruction technique (SART) (Andersen and Kak 1984). Instead of sequentially updating the pixels on a ray-by-ray basis, SART simultaneously applies to a pixel the average of the corrections generated by all rays. This offers a reduction in the amplitude of the salt and pepper noise that is usually present in ART. However, it goes at the expense of the computation time. The SART algorithm is given by

$$
\alpha_{k}^{n+1}=\alpha_{k}^{n}+\frac{w}{W_{+, k}} \frac{p_{i}-\tilde{p}_{i}}{W_{i,+}} W_{i k}
$$

with $W_{+, k}=\sum_{i=1}^{N} W_{i k}, W_{i,+}=\sum_{k=1}^{N} W_{i k}$ and $w$ a relaxation parameter that should be between $\{0,2\}$; we have set it to 1 . Historically, SART is reckoned to the algebraic reconstruction methods. However, recently Jiang and Wang (2003) have shown that SART is a maximum likelihood estimator for the mapping of $\alpha$ in case the error in equation (4) is purely stochastic with a Gaussian distribution.

Some further improvement can be achieved by penalizing unrealistic images. For this, we use the so-called one-steplate algorithm (see Green (1990)). It corrects reconstructed image that suffer from salt and pepper noise. These noisy solutions can be penalized by utilizing a smoothing function. However, this can cause smearing of edges and smoothing of low-contrast details. An algorithm based on the median root prior potential function (suggested first by Alenius and Ruotsalainen (1997)) has been used in our research. It is well suited for removing impulse noise, while preserving edges. In the reconstruction algorithm an extra step is added to equation (5):

$$
\alpha_{\mathrm{OSL}, k}^{n+1}=\frac{1}{1+\beta \frac{\alpha_{\mathrm{OSL}, k}^{n}-\operatorname{Med}\left(\alpha_{\mathrm{OSL}, k}^{n}\right)}{\operatorname{Med}\left(\alpha_{\mathrm{OSL}, k}^{n}\right)}} \alpha_{k}^{n+1}
$$

with $\alpha_{k}^{n}$ being the value of pixel $k$ after the $n$th SART step and $\alpha_{\text {OSL }, k}^{n}$ the same after the $n$th one-step-late correction.

The median filter $\operatorname{Med}\left(\alpha_{k}\right)$ sets the value of the $k$ th pixel equal to the median of the pixel values contained in the $k$ th pixel neighborhood. The definition of this pixel neighborhood is the only parameter of the median filter. We use a $3 \times$ 3 neighborhood; a $5 \times 5$ neighborhood causes too much smearing. The parameter $\beta$ in equation (6) again controls the weight of the prior.

\section{Simulations}

The system we study represents a fluidized bed in which bubbles are moving upwards. Voids are created inside the cross section of the circular fluidized bed. For the particular configuration the forward problem is first solved: for each ray the fraction of solids on the beam is calculated. In the forward problem, the solid particles are assumed to form a uniform quasi-fluid with a constant solids fraction equal to the fraction at packed conditions. For the bubbles, we assume that they are completely empty. This procedure provides the set of ray sums, $\left\{p_{i}\right\}$. Next, noise can be added to the ray sums. The noise is supposed to be Gaussian, with a signal-tonoise ratio of a predescribed percentage of the noise-free ray sum. Obviously, for a complete study of the effects of noise a more rigorous approach is required, based on Monte Carlo simulations, that can deal with scattering effects. Here, we lumped all effects into a single Gaussian noise contribution, allowing only a relatively coarse estimate of the effects of scattering.

Reconstructions of these voids are made with the algorithms describe in section 3 . We have varied a number of parameters:

- $\beta$ : setting $\beta=0$ reduces the solution to the original SART method; for $\beta \neq 0$ we have a regularization according to the median root prior method. We found that the results are rather insensitive to the precise value of $\beta$ if $0.01<\beta<0.5$. The fastest convergence was reached for $\beta=0.1$. Therefore, all reconstructions reported use $\beta=0.1$.

- Number of grid cells, $N_{\text {grid: }}$ this controls the speed and possible resolution of the reconstruction.

- Number of iterations, $N_{\text {iter}}$ : this also influences the speed and possible resolution of the reconstruction. 


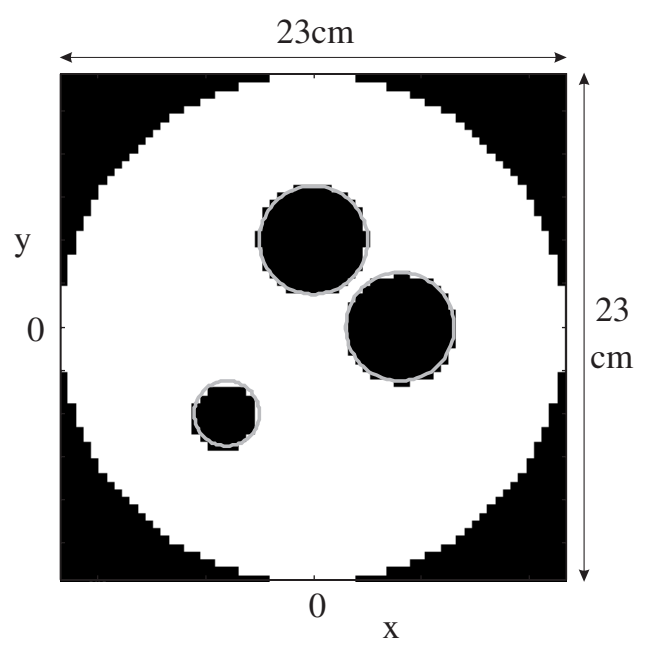

Figure 5. Base case of three voids. The gray circles give the original voids and the black ones the discretized version.

The voids are circular or ellipsoidal in shape, as we expect that a horizontal cut through the bubbles in a fluidized bed will be more or less circular. The grids used are all square in shape. The fluidized bed has a diameter of $23 \mathrm{~cm}$. As mentioned above, our system in the simulations consists of five $\mathrm{x}$-ray sources, each with 30 detectors. The reconstruction runs on a single PC (1.2 GHz). Each iteration step takes about $2.0 \mathrm{~s}$ on a $65 \times 65$ grid, slightly depending on the case.

\subsection{Noiseless case}

The base case is a $23 \mathrm{~cm}$ diameter fluidized bed with a uniform powder fraction. Inside the cross section, that we will reconstruct, three voids in the form of circles are placed. The diameter and coordinates of their center of gravity $\{x, y, d\}$ are $\{4,0,5\},\{0,4,5\}$ and $\{-4,-4,3\}$, respectively (all in $\mathrm{cm}$; the origin of the coordinate system coincides with the axis of the fluidized bed). The void fraction $\alpha$ is stretched from 1, i.e. powder only, to 0 which denotes no powder at all, i.e. air only. Note that in all figures presented, we have reversed the pixel values: black $(=0)$ indicates air, white $(=1)$ powder.

We use as a base grid for reconstruction, a square grid of $65 \times 65$ pixels, with a pixel size of $3.54 \mathrm{~mm}$. The base case is shown in figure 5 . For the calculation of the ray sums we have used the discretized version of the voids. For this discretization a threshold value of 0.5 is used. If $50 \%$ or more of a pixel is part of a void, its value is set to 0 . Otherwise its value is 1 . Note that the gap between the two large circles has a width of the diagonal of one pixel, i.e. $5 \mathrm{~mm}$. The voids have a size of 160, 160, 54 (in pixel ${ }^{2}$ ).

For the reconstruction, the error is defined as the difference between the original and the reconstructed images:

$$
\text { error }=\frac{\sum_{i=1}^{N_{\text {grid }}} \sum_{j=1}^{N_{\text {grid }}}\left|\alpha_{i j}^{\text {new }}-\alpha_{i j}^{\text {org }}\right|}{N_{\text {bed }} * N_{\text {bed }}}
$$

with $N_{\text {bed }}$ being the number of pixels inside the actual bed.

The reconstruction is started with as initial guess a fluidized bed completely filled with powder. Note that the pixels outside the fluidized bed are set to zero by definition.

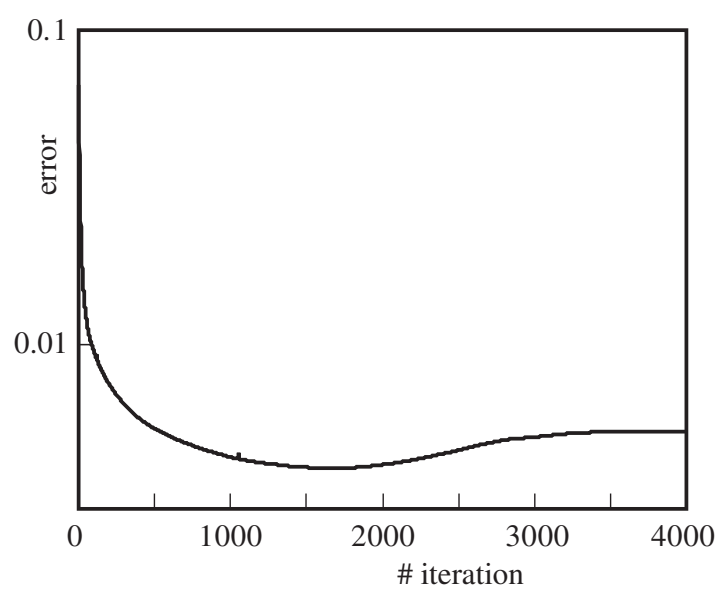

Figure 6. Error of the reconstructed image as a function of the number of iterations.

After each new iteration these pixels are set to zero again. Thus, they do not influence the error. Furthermore, a single pixel flipping from 0 to 1 gives a change in the error of $2.9 \times 10^{-4}$. Smaller values can be achieved as the pixel values may vary continuously from 0 to 1 . In figure 6 , the error is given as a function of the number of iterations.

With increasing number of iterations, the image becomes closer to the original one. In figure 7 , several reconstructed images are shown. In all cases the gap between the two large voids is well recovered. In the binary images, an object is defined as a collection of connected foreground pixels, using an 8-connectivity scheme. Hence, the minimum size of the gap implies that the two objects cannot touch. The more iterations are used, the more is the noise in the image reduced. The resolution, in terms of separation of objects, is thus better than $5 \mathrm{~mm}$. Note that the error goes slightly up after approximately 2000 iterations. It is not clear why. However, the effect on the reconstructed images is quite small: it concerns only a few pixels.

A thresholding on the pixels of 0.5 is used to turn the raw reconstructed images into binary ones: if a pixel value is larger than the threshold its binary value is set to 1 , if it is smaller to 0 . In table 4, the area (in pixel ${ }^{2}$ ) and the center of gravity (in $\mathrm{cm}$ ) of the reconstructed voids are given. The area is found from the images by simply calculating the number of pixels that form the object in the binary image. The center of gravity is also obtained from the binary object as the average of all $x$ and $y$-coordinates of the object pixels. It is found that for the noiseless case, the area and the position of the voids are well reconstructed. It is found that 400 iterations are sufficient.

\subsection{Influence of noise}

The above reconstruction started from noiseless data. In this section, Gaussian-distributed noise is added to the ray sums, according to $p_{i} \rightarrow p_{i} \cdot\left(1+A \cdot n_{i}\right)$ with $n_{i}$ being a random noise contribution with a Gaussian distribution of zero mean and a standard deviation equal to $1 . A$ is the amplitude of the noise and is given as a percentage. In table 5, reconstructions of the base case are compared when $1 \%, 2 \%, 4 \%$ or $8 \%$ of noise 


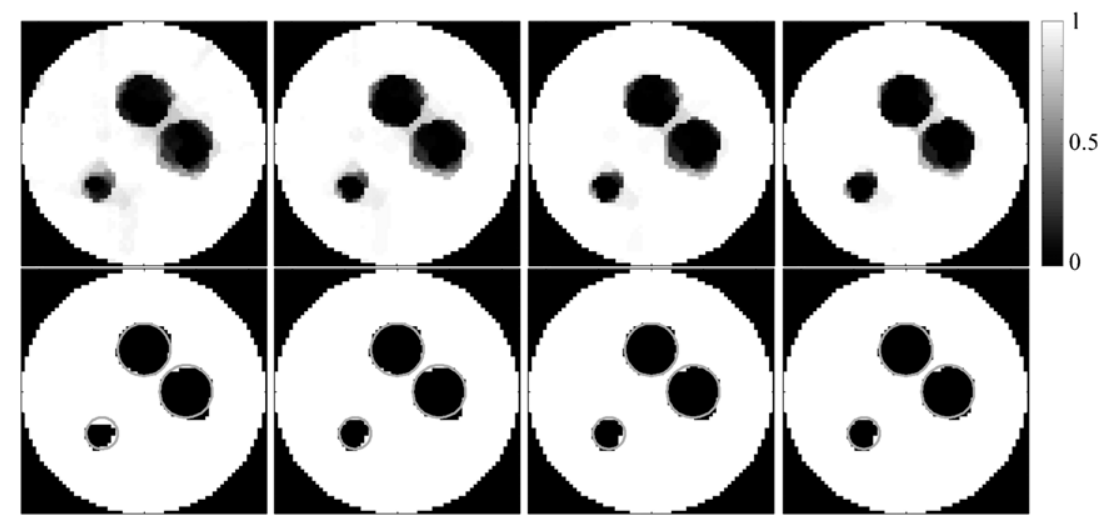

Figure 7. Comparison of the reconstructed images. Top: raw images, bottom: thresholded images (threshold value 0.5). From left to right after 100, $200,400,800$ iterations, respectively.

Table 4. Base case: area in pixel ${ }^{2}$ and coordinates of center of gravity of objects. The original image is denoted by 'org' and the reconstructed binary images by 'b' followed by the number of iterations to get them.

\begin{tabular}{llllllllll}
\hline & Area $\left(\mathrm{pixel}^{2}\right)$ & $x_{c}(\mathrm{~cm})$ & $y_{c}(\mathrm{~cm})$ & Area $\left(\mathrm{pixel}^{2}\right)$ & $x_{c}(\mathrm{~cm})$ & $y_{c}(\mathrm{~cm})$ & Area $_{\left(\mathrm{pixel}^{2}\right)}$ & $x_{c}(\mathrm{~cm})$ & $y_{c}(\mathrm{~cm})$ \\
\hline Org & 54 & -4.03 & -4.03 & 160 & 0.00 & 4.04 & 160 & 4.04 & 0.00 \\
b100 & 42 & -4.14 & -4.08 & 162 & 0.09 & 4.12 & 162 & 4.12 & -0.13 \\
b200 & 49 & -4.08 & -3.96 & 159 & 0.04 & 4.10 & 162 & 4.12 & -0.13 \\
b400 & 51 & -4.05 & -4.00 & 162 & 0.02 & 4.06 & 162 & 4.09 & -0.07 \\
b800 & 51 & -4.05 & -4.00 & 164 & 0.05 & 4.05 & 161 & 4.08 & -0.05 \\
\hline
\end{tabular}

Table 5. Influence of noise: area and coordinates of center of gravity of objects. The original image is denoted by 'org'; the reconstructed binary images by 'n' followed by the percentage of noise added.

\begin{tabular}{lllllrlllr}
\hline & $\begin{array}{l}\text { Area } \\
\left(\mathrm{pixel}^{2}\right)\end{array}$ & $\begin{array}{l}x_{c} \\
(\mathrm{~cm})\end{array}$ & $\begin{array}{l}y_{c} \\
(\mathrm{~cm})\end{array}$ & $\begin{array}{l}\text { Area } \\
\left(\mathrm{pixel}^{2}\right)\end{array}$ & $\begin{array}{l}x_{c} \\
(\mathrm{~cm})\end{array}$ & $\begin{array}{l}y_{c} \\
(\mathrm{~cm})\end{array}$ & $\begin{array}{l}\text { Area } \\
\left(\mathrm{pixel}^{2}\right)\end{array}$ & $\begin{array}{l}x_{c} \\
(\mathrm{~cm})\end{array}$ & $\begin{array}{r}y_{c} \\
(\mathrm{~cm})\end{array}$ \\
\hline $\mathrm{Org}$ & 54 & -4.03 & -4.03 & 160 & 0.00 & 4.04 & 160 & 4.04 & 0.00 \\
$\mathrm{n} 1 \%$ & 47.5 & -4.10 & -3.98 & 164 & 0.03 & 4.08 & 156 & 4.07 & -0.07 \\
& \pm 2.3 & \pm 0.04 & \pm 0.05 & \pm 4 & \pm 0.06 & \pm 0.03 & \pm 5 & \pm 0.05 & \pm 0.05 \\
$\mathrm{n} 2 \%$ & 46.7 & -4.16 & -3.99 & 162 & 0.05 & 4.06 & 157 & 4.01 & -0.09 \\
& \pm 4.3 & \pm 0.09 & \pm 0.08 & \pm 7 & \pm 0.07 & \pm 0.06 & \pm 7 & \pm 0.08 & \pm 0.06 \\
$\mathrm{n} 4 \%$ & 42.4 & -4.20 & -3.96 & 162 & 0.13 & 4.05 & 155 & 4.01 & -0.08 \\
& \pm 6.1 & \pm 0.19 & \pm 0.17 & \pm 9 & \pm 0.09 & \pm 0.10 & \pm 10 & \pm 0.13 & \pm 0.12 \\
$\mathrm{n} 8 \%$ & 16 & -4.60 & -3.72 & 146 & 0.26 & 3.56 & 143 & 4.28 & -0.62 \\
\hline
\end{tabular}

is added. For each noise level, 40 different reconstructions are used. In all cases 400 iterations are used in a reconstruction. The results, i.e. the mean and standard deviation, are given in table 5 . Of the reconstructed voids with $1 \%$ noise in $2.5 \%$ of the 40 cases, with $2 \%$ noise in $18 \%$, with $4 \%$ noise in $37 \%$ of the 40 cases the gap between the two large voids was partly closed. Nevertheless, it was clear for the eye that two objects were present in the reconstructed images.

From the table, we see that, obviously, the reconstruction deteriorates with increasing noise. For a proper reconstruction the noise level should be kept at a level of $1 \%$ or less. The spatial resolution with $1 \%$ noise still allows us to discriminate between objects that are spaced about one grid cell apart. A consequence of the noise is that the area of the smaller object is in the reconstructions smaller for higher noise levels. This is caused by a smearing of the object over adjacent pixels. As we kept the threshold constant, the area of the thresholded object will drop. More advanced image analysis techniques, in which the threshold is based on the actual image, will probably improve the reconstructions.

\subsection{Grid size}

The influence of the grid size is investigated for the noiseless case. For the same voids, we varied the grid size from $25 \times 25,35 \times 35, \ldots, 65 \times 65$. For each grid, a reconstruction is made, based on 400 iterations. The area and location of the voids are calculated using a fixed threshold value of 0.5 . The area is given in table 6 , in $\mathrm{cm}^{2}$ rather than in pixel $^{2}$ to facilitate comparison. The gap between the two original voids is $0.65 \mathrm{~cm}$. For the grid of $65 \times 65$ pixels, the pixel size is $0.35 \mathrm{~cm}$ (the pixel diagonal $0.50 \mathrm{~cm}$ ) and the two large voids are separated by at least one pixel. For the grids $25 \times 25$ and $35 \times 35$ the pixel size is larger than the separation distance between the voids, and smearing potentially causes the two large voids to merge into one object. This is indeed found: for the $35 \times 35$ grid the two voids are 'glued together' but clearly two objects can be distinguished. For the $25 \times 25$ grid, this is no longer the case. Here, the two voids have merged into one elongated object. Without a priori knowledge this object cannot be separated into two. 

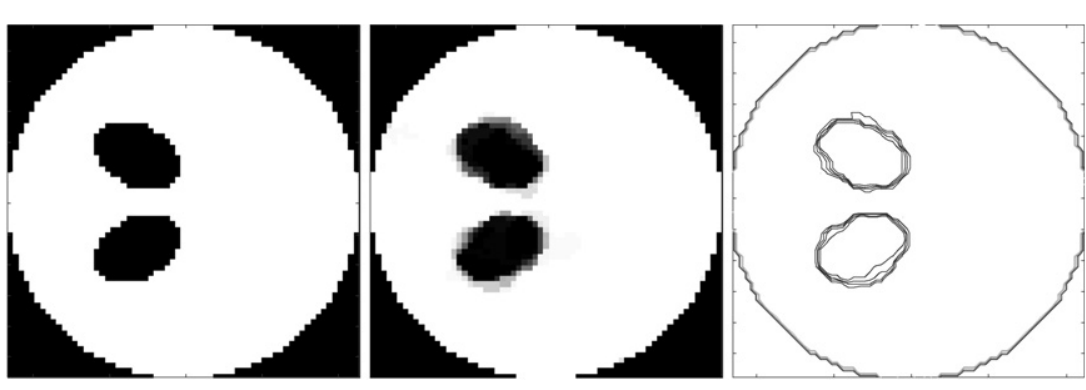

Figure 8. Reconstruction of two ellipses $(65 \times 65$ grid, 400 iterations). Left: original discretized voids, middle: reconstructed image, right: contour plot of reconstructed images (contour lines indicate gray levels of 0.2, 0.4, 0.6 and 0.8).

Table 6. Base case: area in $\mathrm{cm}^{2}$ and coordinates of center of gravity of objects. The reconstructed binary images are denoted by ' $\mathrm{g}$ ' followed by the grid size.

\begin{tabular}{llllllllll}
\hline & $\begin{array}{l}\text { Area } \\
\left(\mathrm{cm}^{2}\right)\end{array}$ & $\begin{array}{l}x_{c} \\
(\mathrm{~cm})\end{array}$ & $\begin{array}{l}y_{c} \\
(\mathrm{~cm})\end{array}$ & $\begin{array}{l}\text { Area } \\
\left(\mathrm{cm}^{2}\right)\end{array}$ & $\begin{array}{l}x_{c} \\
(\mathrm{~cm})\end{array}$ & $\begin{array}{l}y_{c} \\
(\mathrm{~cm})\end{array}$ & $\begin{array}{l}\text { Area } \\
\left(\mathrm{cm}^{2}\right)\end{array}$ & $\begin{array}{l}x_{c} \\
(\mathrm{~cm})\end{array}$ & $\begin{array}{l}y_{c} \\
(\mathrm{~cm})\end{array}$ \\
\hline Org & 7.1 & -4.00 & -4.00 & 19.6 & 0 & 4.00 & 19.6 & 4.00 & 0 \\
$\mathrm{~g} 25$ & 5.2 & -4.14 & -3.68 & 42.3 & 2.06 & 1.84 & & & \\
$\mathrm{~g} 35$ & 6.9 & -4.27 & -3.94 & 20.7 & -0.12 & 3.82 & 20.7 & 3.82 & 0.12 \\
$\mathrm{~g} 45$ & 6.3 & -4.02 & -3.92 & 18.0 & 0.00 & 4.09 & 18.3 & 4.03 & -0.04 \\
$\mathrm{~g} 55$ & 6.0 & -4.20 & -4.03 & 18.2 & 0.04 & 3.97 & 19.2 & 4.06 & -0.03 \\
$\mathrm{~g} 65$ & 6.4 & -4.05 & -4.00 & 20.3 & 0.02 & 4.06 & 20.3 & 4.09 & -0.07 \\
\hline
\end{tabular}

The area of the original circular voids is $7.1 \mathrm{~cm}^{2}$ and $19.6 \mathrm{~cm}^{2}$ for the small and two larger voids, respectively. Obviously, due to the discretization and thresholding, the voids on the actual grids are smaller.

The results for the $65 \times 65$ grid are the best, but the difference with the $55 \times 55$ one is small. The $25 \times 25$ grid is insufficient for decent reconstructions.

\subsection{Elliptical voids}

The cut of the measuring plane through the voids in an actual experiment is not necessarily a circle. In this subsection, two elliptically shaped voids are used. These voids have major and minor half-axis $a=3 \mathrm{~cm}$ and $b=2 \mathrm{~cm}$, hence an area of $18.9 \mathrm{~cm}^{2}$. They are shifted over a vector $\left\{x_{s}, y_{s}\right\}$ with respect to the origin and their orientation is rotated over an angle $\theta$. Figure 8 shows the original and reconstructed ellipses for the noiseless case, with $\left\{x_{s}, y_{s}\right\}=\{-3 \mathrm{~cm}, \pm 3 \mathrm{~cm}\}$ and $\theta= \pm \pi / 6$. The minimum distance of the two ellipses is about $1.4 \mathrm{~cm}$, i.e. about 4 pixels. Also a contour plot is given. The ellipses are correctly reconstructed. Using a threshold value of 0.5 the reconstructed image is made binary. From this binary image, the ellipse area and center of gravity are computed. For the top ellipse we find $A=19.6 \mathrm{~cm}^{2}, x_{c}=-2.99 \mathrm{~cm}, y_{c}=$ $3.06 \mathrm{~cm}$. For the bottom ellipse these numbers are $18.4 \mathrm{~cm}^{2}$, $-3.01 \mathrm{~cm},-2.97 \mathrm{~cm}$, respectively.

We tested several other angles: no new peculiarities are found. Like in the case of the circular voids, noise starts blurring the images. Moreover, if the objects are separated a distance smaller than 2 pixels they generally merge into a single object. The angle of the major axis of the ellipse with the horizontal in the images has been changed up to $60^{\circ}$ and the position of the two ellipses in the bed has been varied. No particular dependence on these two variables was found. We used two smaller ellipses ( $a=2 \mathrm{~cm}, b=1 \mathrm{~cm})$. These can be reconstructed correctly up to a noise level of $2 \%$.

\section{Experiments}

\subsection{Calibration}

The reconstruction uses as starting point the measured attenuation of the x-ray beams. A calibration procedure relates the measured attenuation to the distance the x-ray beam travels through the powder mass. The calibration curve is obtained by placing a known length or powder mass in each beam and measuring the attenuation. An example is given in figure 9. This calibration curve is accurately described by $A_{\text {cal }}+B_{\text {cal }} \exp \left(-x / C_{\text {cal }}\right)$, with $x$ being the distance traveled by the beam through the powder phase. For each detector, an individual calibration curve is obtained. Note that the calibration curve in figure 9 is for the central detector and covers only a range from $9 \mathrm{~cm}$ to $26 \mathrm{~cm}$ of polystyrene path length. This is done as the expected path length will not drop below $9 \mathrm{~cm}$. Limiting the range allows for a better exponential fit. This is a practical argument. For calibration over the full range of possible path lengths, a different fitting function or even a look-up table can be used. The exponential fit function has no significance in the process. Indeed, for the detectors receiving the $\mathrm{x}$-ray from the side of the bed a different range is used for the calibration.

\subsection{Experimental procedure}

For all experiments, the maximum tube voltage setting of $150 \mathrm{kV}$ has been used. The photon rate of the x-ray source is controlled by the tube current. For the current collimator configuration, signal amplification and object, it has been determined that the minimum tube current setting of $10 \mathrm{~mA}$ 


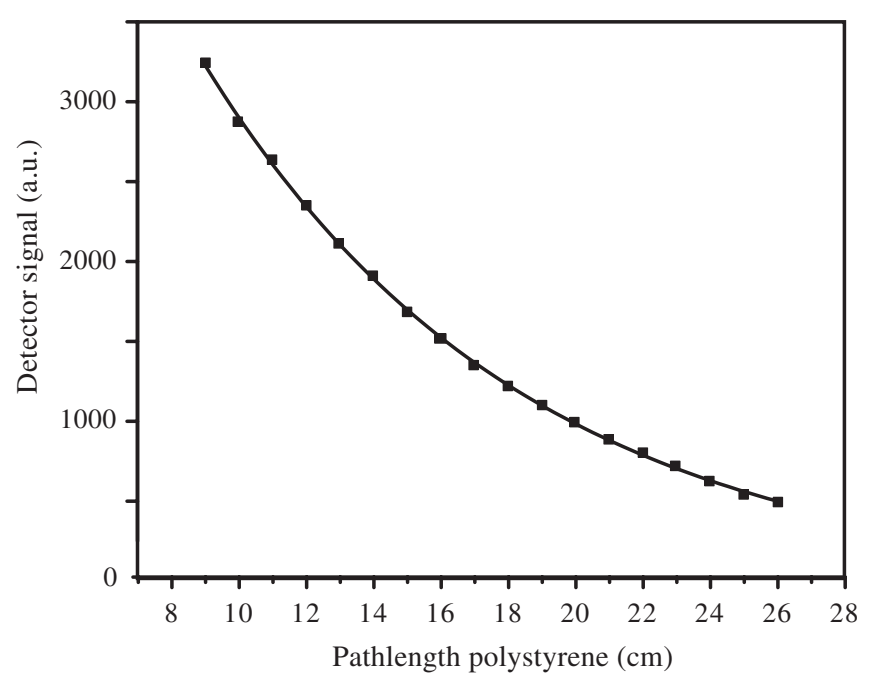

Figure 9. Calibration curve of a detector.

leads to optimal use of the available dynamic range for the detectors.

The scanner system is controlled from one master PC. Custom-made software has been developed for the automation of tomographic measurements. Each measurement consists of a sequence (see below) of commands and trigger signals sent from the master PC to the different functionalities. This measurement program is based on the simulation of a 5 -source setup, which means that the measurement sequence is repeated five times. For each measurement the rotary table is rotated $72^{\circ}$ in the clockwise direction. The specific steps for an experiment are as follows:

(1) Move rotary table to predefined position.

(2) Load x-ray tube (trigger).

(3) Start data acquisition (trigger).

(4) Start X-ray pulse of predefined duration (trigger).

\subsection{Pre-processing of tomographic data}

Before the tomographic data are fed to the actual reconstruction algorithm, some pre-processing takes place:

(1) The signal values of both the void measurement and filled vessel reference measurement (including the void cylinders filled with powder) are converted to equivalent lengths of packed polystyrene, using the detector calibration. Note that the calculated lengths include the thickness of the perspex walls of the vessel and the walls of the cylinders, expressed in equivalent lengths of packed polystyrene.

(2) The converted void measurement data are subtracted from the converted filled vessel reference data. In this way, the contribution of the vessel walls and cylinder walls is effectively removed.

(3) The calculated difference data file is in turn subtracted from a data file containing the calculated lengths of polystyrene corresponding to a filled vessel. Now the data represent the total length polystyrene for each line. These data are fed to the reconstruction algorithm.

In figure 10, a measurement through two air-filled cylinders positioned in the fluidized bed is shown. The large cylinder has a diameter of $5.1 \mathrm{~cm}$. Its center of gravity coordinates are $\left\{x_{c}, y_{c}\right\}=\{-16.7 \mathrm{~mm},-52.1 \mathrm{~mm}\} . x$ denotes the horizontal coordinate and $y$ the vertical one with the origin coinciding with the axis of the fluidized bed, see figure 10. The smaller cylinder has a diameter of $2.2 \mathrm{~cm}$ (with $\left\{x_{c}, y_{c}\right\}=\{-7.2 \mathrm{~mm},-9.0 \mathrm{~mm}\}$ ). The separation distance between the two is $1 \mathrm{~cm}$. The gray line indicates the center line of one of the fan-beam configurations. The vertical axis of the measured data is given in 'cm air'. This denotes the length a given beam moves through air in the bed. Note that this comprises air in the cylinder (if the beam crosses a cylinder) but also the air in between the powder particles. Furthermore, it should be kept in mind that each beam moves over a different length through the fluidized bed. Nevertheless, the measured

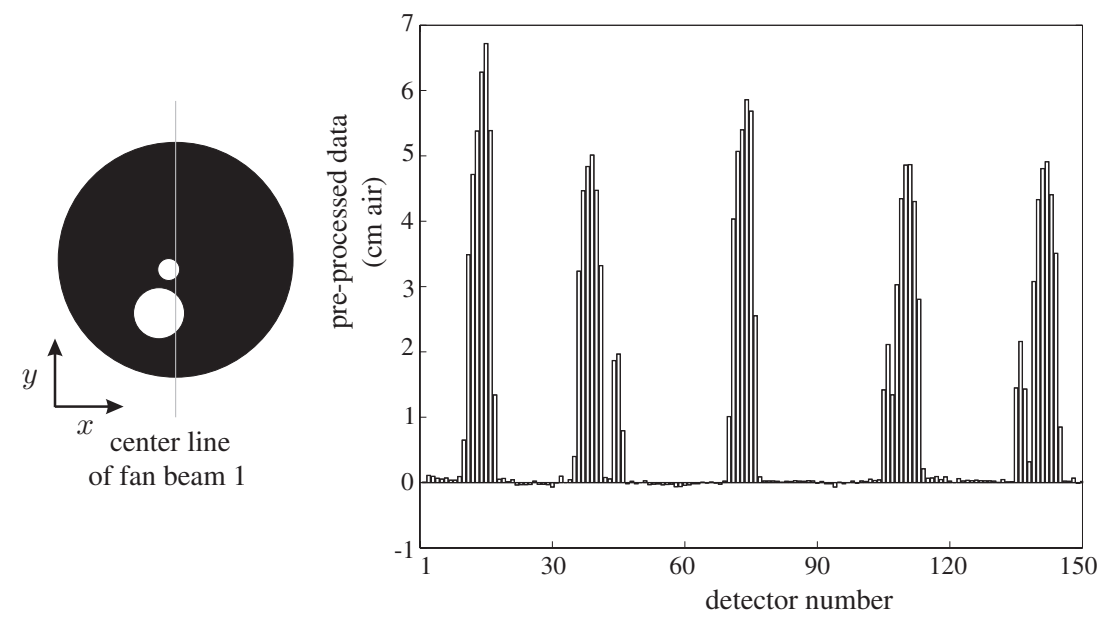

Figure 10. Two bubbles (left), the gray line indicates the center line of one of the fan-beam positions. Measured data, in terms of length of air on the beams, are shown on the right. 

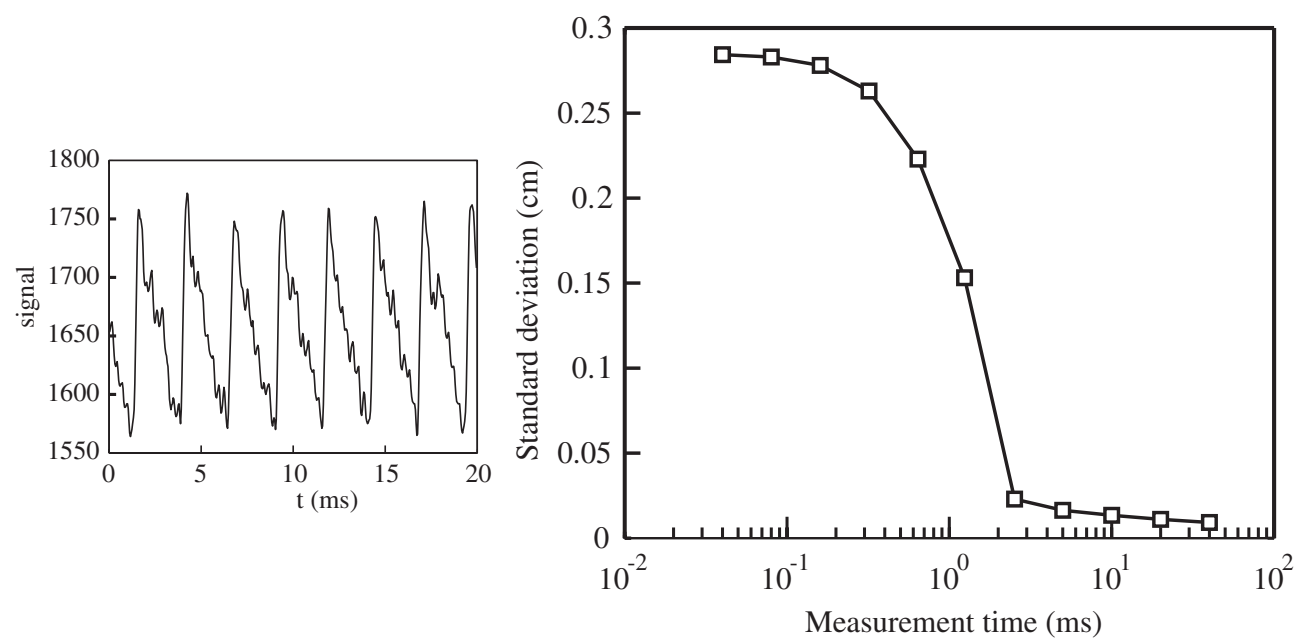

Figure 11. Left: the effect of voltage ripple on detector signal. Right: the standard deviation of the mean signal values as a function of the measurement time (number of samples in a subset).

data clearly reveal the two voids in the fluidized bed: see the 2nd and 5th fan beam where both a big peak and a small peak are measured.

\subsection{Measurement error and sample time}

An important feature of tomographic measuring techniques is the measurement time. This is one of the bottlenecks for nuclear techniques. For measurements on static voids, detector signals can be averaged over relatively long times. This reduces the effect of stochastic errors. To determine the real time resolution of the scanner system, the behavior of the error is considered as a function of the frame rate. This has been done by analyzing a data set of a single detector. The data set consists of 18000 data points of a static object measured at a sampling frequency of $25 \mathrm{kHz}:\left\{Y_{i}\right\}$ with $i=1, \ldots, N$ and $N=18000$. The data set has an average value $\bar{Y}=\frac{1}{N} \sum_{i=1}^{N} Y_{i}$.

The full data set has been divided in $K$ subsets of equal size, each subset contains $M$ data points: $\left\{X_{j}\right\}_{K}$ with $j=1, \ldots, M$ and $K=\operatorname{int}(N / M)$. Each subset has a mean $\bar{X}_{K}=\frac{1}{M} \sum_{j=1}^{M}\left\{X_{j}\right\}_{K}$. The number of data points in a subset, $M$, is equivalent to the measuring time for a single tomogram. The uncertainty due to the finite measuring time can be estimated by inspecting the uncertainty in the mean of a subset. The standard deviation of this mean is calculated from the unbiased variance:

$$
\operatorname{Var}\left(\left[\bar{X}_{1} \cdots \bar{X}_{N}\right]\right)=\frac{1}{N-1} \sum_{i=1}^{N}\left(\bar{X}_{i}-\bar{Y}\right)^{2} .
$$

The results for a number of subset sizes, with the standard deviation given in terms of centimeters packed polystyrene, are shown in figure 11. The number of points in each subset has been translated to a measurement time, $\Delta t$, by using the data rate of $25000 \mathrm{~Hz}: \Delta t=M / 25 \mathrm{kHz}$. The five points with the low standard deviation in the graph correspond to averages based on 62, 125, 250, 500 and 1000 samples, respectively.

It is clear from figure 11 that the standard deviation increases quickly for measurement times smaller than
$2.5 \mathrm{~ms}$. This is caused by a voltage ripple on the high voltage unit, see figure 11 (left graph). The point at which the 'kink' occurs corresponds to exactly one period of the voltage ripple. This limits the accuracy of the tomograph for the moment. Either the ripple needs to be reduced by a better power supply or the measured data are corrected, by measuring the phase of the voltage ripple. For the present equipment, the measuring error can be kept below $1 \%$ (i.e. $0.2 \mathrm{~cm}$ over $23 \mathrm{~cm}$ diameter) for measuring times of $2.5 \mathrm{~ms}$ or larger. The present setup, thus, in principle allows accurate tomography with a frame rate of 400 frames $\mathrm{s}^{-1}$. Note that for an actual scanner, with multiple sources, scattering from $\mathrm{x}$-rays from one source on the detectors of another source may deteriorate the reconstructions significantly. Most likely, collimation of the detectors will be necessary. As an alternative, the sources could fire xrays not simultaneously, but sequentially. This way scattering from one source to another set of detectors is excluded. This, however, requires a fast system: the object under study should be 'frozen' during this measuring sequence.

\section{Experimental results}

\subsection{Static voids}

The measured data are reconstructed on a $65 \times 65$ pixel based reconstruction grid (spatial resolution $3.5 \mathrm{~mm}$ ), using the SART algorithm with regularization described above. The result is given in figure 12. Both cylinders are clearly identified.

By using a threshold value, the gray value figures are turned into binary ones, from which the size and location of the two voids are calculated. In table 7 , the results for various threshold values are given. We find that the size and position of the large void are rather insensitive to the threshold used. This is not true for the smaller void. Here, the area depends on the choice of the threshold value. This is obvious from figure 12: the gray values corresponding to the small cylinder span only a small range. Here more advanced image analysis 


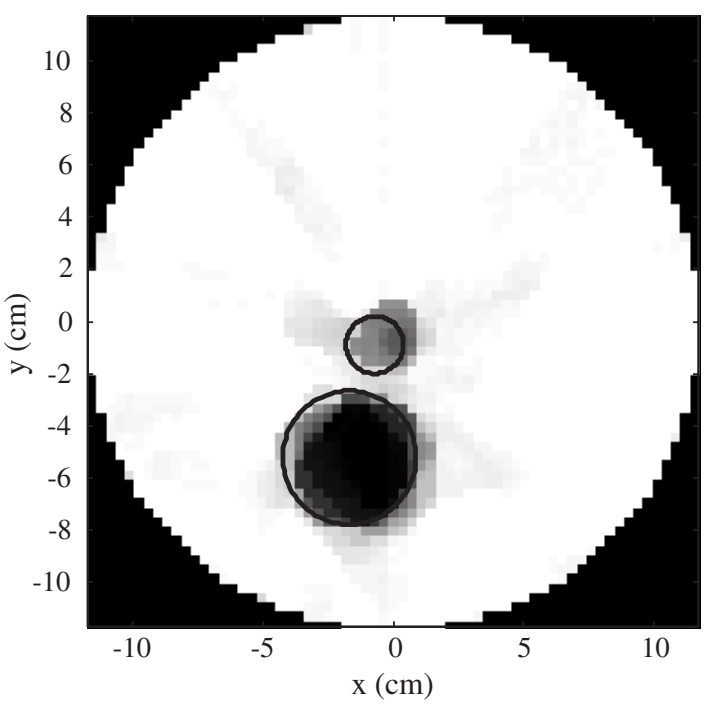

Figure 12. Reconstructions of the two cylinder case. The two circles denote the true position and size of the cylinders.

Table 7. Characteristics of the two voids.

\begin{tabular}{|c|c|c|c|c|c|}
\hline \multirow[b]{2}{*}{ Void } & \multirow[b]{2}{*}{ Threshold } & \multicolumn{2}{|c|}{ Area $\left(\mathrm{cm}^{2}\right)$} & \multirow{2}{*}{$\begin{array}{l}x_{c}(\mathrm{~cm}) \\
\text { Recon }\end{array}$} & \multirow{2}{*}{$\begin{array}{l}y_{c}(\mathrm{~cm}) \\
\text { Recon }\end{array}$} \\
\hline & & Org & Recon & & \\
\hline 1 & 0.45 & 3.8 & 1.0 & 0.2 & -0.7 \\
\hline 2 & 0.45 & 20.4 & 18.3 & -1.3 & -5.2 \\
\hline 1 & 0.5 & 3.8 & 1.9 & 0.2 & -0.7 \\
\hline 2 & 0.5 & 20.4 & 18.5 & -1.3 & -5.2 \\
\hline 1 & 0.55 & 3.8 & 3.6 & -0.1 & -0.5 \\
\hline 2 & 0.55 & 20.4 & 19.3 & -1.4 & -5.2 \\
\hline 1 & 0.6 & 3.8 & 4.6 & -0.2 & -0.5 \\
\hline 2 & 0.6 & 20.4 & 20.2 & -1.3 & -5.2 \\
\hline 1 & 0.65 & 3.8 & 4.9 & -0.2 & -0.5 \\
\hline 2 & 0.65 & 20.4 & 21.4 & -1.3 & -5.2 \\
\hline 1 & 0.75 & 3.8 & 5.1 & -0.2 & -0.5 \\
\hline 2 & 0.75 & 20.4 & 23.5 & -1.3 & -5.2 \\
\hline
\end{tabular}

techniques will be required to find objective measures of this void.

In all cases, a clear separation between the two objects is found. It is no problem to identify two objects.

A second case is measured and reconstructed, consisting of two $5.1 \mathrm{~cm}$ diameter and two $2.2 \mathrm{~cm}$ diameter cylinders, placed on the $x$ - and $y$-axes, see figure 13. A threshold value of 0.5 is used to find the area and location of the voids. From the area, $A$, an equivalent diameter is calculated as $D_{\text {eq }}=\sqrt{\frac{4}{\pi} A}$. Our findings are summarized in table 8. Again we see that we reproduce the voids quite accurately and that we tend to underestimate the smaller ones.

\subsection{Moving voids}

Two different moving voids have been used. These voids are moved upwards through the bed at a constant speed. Each experiment is repeated five times, with a $72^{\circ}$ rotation of the

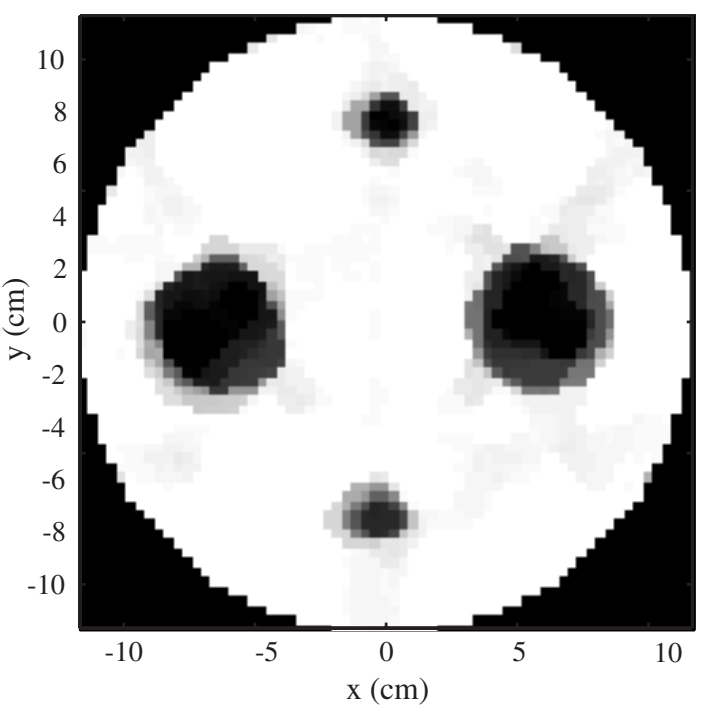

Figure 13. Reconstructions of the four cylinder case.

Table 8. Characteristics of the four voids.

\begin{tabular}{lll}
\hline & \multicolumn{2}{c}{$D_{\text {eq }}(\mathrm{cm})$} \\
\cline { 2 - 3 } Void & Org & Recon \\
\hline Top & 2.2 & 2.0 \\
Right & 5.1 & 5.3 \\
Bottom & 2.2 & 1.9 \\
Left & 5.1 & 5.0 \\
\hline
\end{tabular}

bed for each measurement. This way a 5-source scan is mimicked. A trigger is used to mark a starting position, so all five measurements start with the moving void at exactly the same position. This is obviously needed for a proper tomographic reconstruction.

Moving void 1 is an air-filled cylinder with an outer diameter of $51 \mathrm{~mm}$ and a height of $42 \mathrm{~mm}$. Above and below it is a $40 \mathrm{~mm}$ high chamber filled with the same powder as the fluidized bed. The void is moving through a thin perspex tube, that is placed off center. The cylinder is mounted at the end of a rod, that is pulled upwards at a predefined fixed velocity. See figure 14 for a sketch. Void 2 is an egg made of an air-filled, thin expanded polystyrene shell. It has a diameter of $46 \mathrm{~mm}$ and a height of $66.5 \mathrm{~mm}$.

Moving cylinder. Figure 15 shows the response of one of the detectors when the cylindrical void passes (rise velocity $20 \mathrm{~cm} \mathrm{~s}^{-1}$ ). Different points are marked in the graph. At point 1 , the $\mathrm{x}$-ray tomograph starts measuring. The top cylinder filled with powder is then already cutting through the measuring plane. At point 2, the perspex disc separating the upper powder chamber from the empty part of the void moves into the field of view of the detector and the signal drops. The signal reaches a sharp minimum when the disc with thickness $4 \mathrm{~mm}$ is completely inside the field of view of the detector. We estimate that based on geometrical arguments the field of view has a thickness of $4 \mathrm{~mm}$. This seems to be confirmed by the experiments. However, from the length of the time interval of $\operatorname{dip} 3(0.057 \mathrm{~s})$ and the velocity of the cylinder $\left(20 \mathrm{~cm} \mathrm{~s}^{-1}\right) \mathrm{we}$ find that the distance over which the plastic disc is displaced 

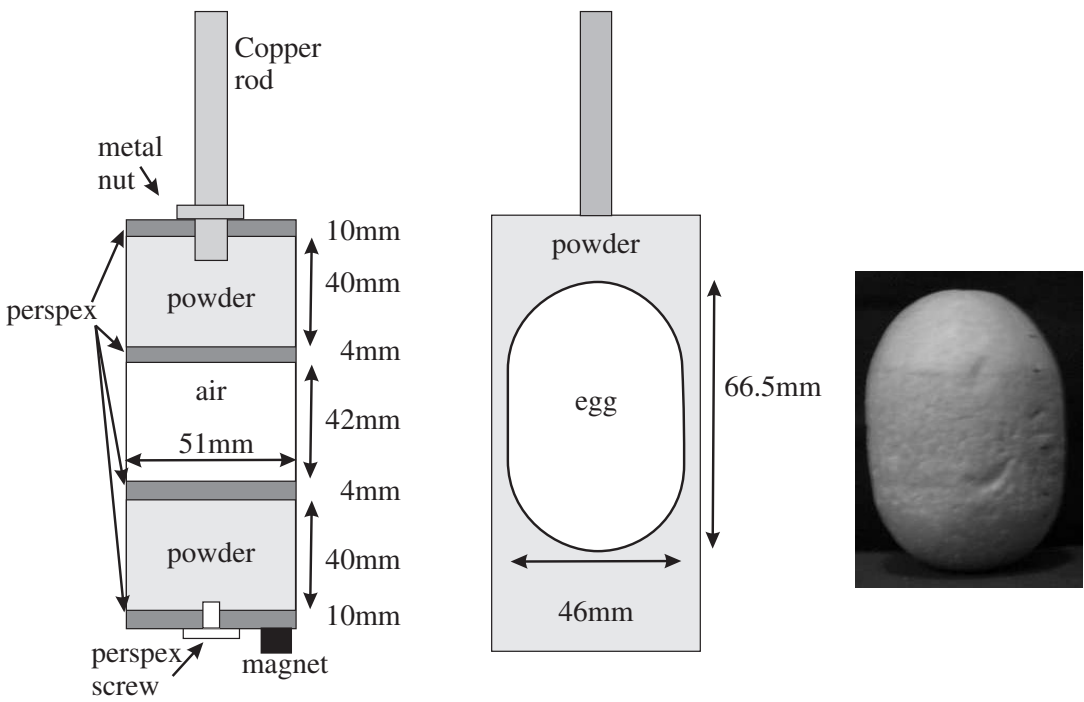

Figure 14. Sketch of the moving voids used. Left: cylinder, middle: egg, right photo of egg.

from entering to leaving the measurement plane is $11 \mathrm{~mm}$. Combining this with the thickness of the disc (4 mm), we have that in practice the measuring plane has a thickness of $7 \mathrm{~mm}$. This is about $40 \%$ more than estimate from the geometry. This point needs further attention. Note that the copper rod used to pull to object up also scatters the x-rays.

At point 4, the air pocket is detected and the output is maximal. Dip 5 is again a perspex disc. At point 6, the output level is slightly higher than the powder-filled bottom chamber (point 7) would give. This is due to the fact that the lower chamber is not $100 \%$ filled with powder: a small air gap is present. This is detected by the system; it shows the level of details that can be reached. Points 8 and 9 mark the perspex screw and the magnet (used for triggering the x-ray system), respectively (see figure 14). Finally, ten indicates that after the passage of the void the powder has to flow into the space left behind by the void. This takes some time as can be seen: initially a higher output level is found before (at 11) the level has returned to 'all powder'. It should be noted that the data are taken from a measuring plane of finite thickness.

Moving egg. In figure 16 a typical raw signal is given for the passage of the egg at a velocity of $10 \mathrm{~cm} \mathrm{~s}^{-1}$. The egg is contained in a thin walled cylinder filled with the polystyrene powder. The entire cylinder is moved upwards through the fluid bed at fixed velocity.

Again the signal is averaged using a window of $5 \mathrm{~ms}$ to remove the voltage ripple. These filtered signals are fed to the reconstruction algorithm. A sequence of images with the passing egg at various stages is generated. An example is given in figure 17, showing the movement of the top of the egg into the measuring plane.

These images are subjected to a threshold to determine which pixel is part of the egg. The diameter of the reconstructed cut through the egg is calculated from the area, $A_{c}: D=\sqrt{\frac{4}{\pi} A_{c}}$. The area itself is calculated as the weighted

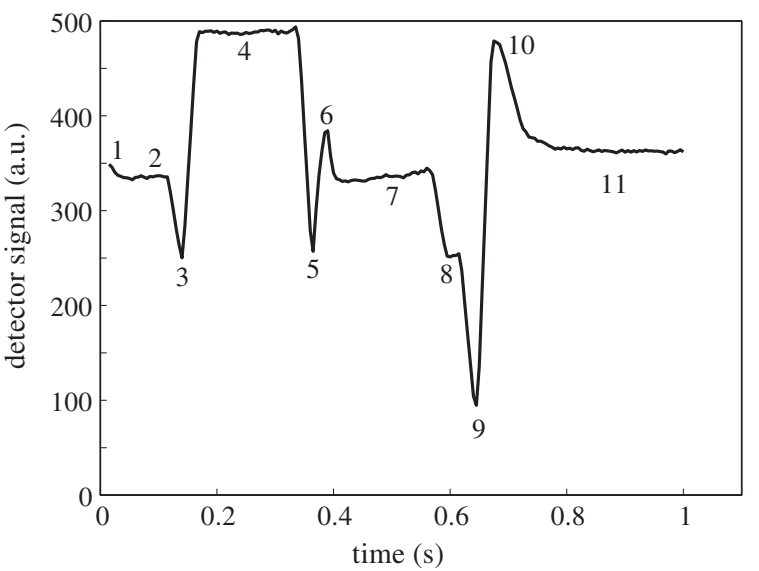

Figure 15. Typical detector signal for a vertical velocity of $20 \mathrm{~cm} \mathrm{~s}^{-1}$ of the cylinder. The signal is obtained by averaging the raw signal over 128 points, i.e. a sampling time of $5 \mathrm{~ms}$ is achieved.

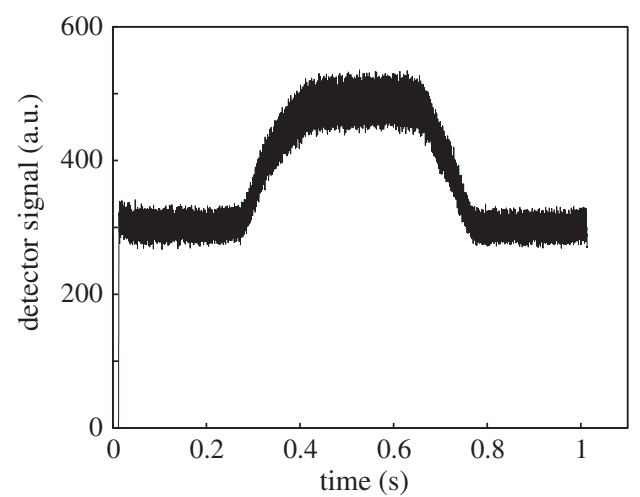

Figure 16. Typical raw signal for the moving egg $\left(10 \mathrm{~cm} \mathrm{~s}^{-1}\right)$.

sum of the pixels in the fluidized bed. The weighing factors used are the gray values, $\left\{g_{i}\right\}$, of the pixels:

$$
A_{c}=\frac{\sum_{i}^{N} g_{i}}{N} A_{\text {fluidbed }}
$$




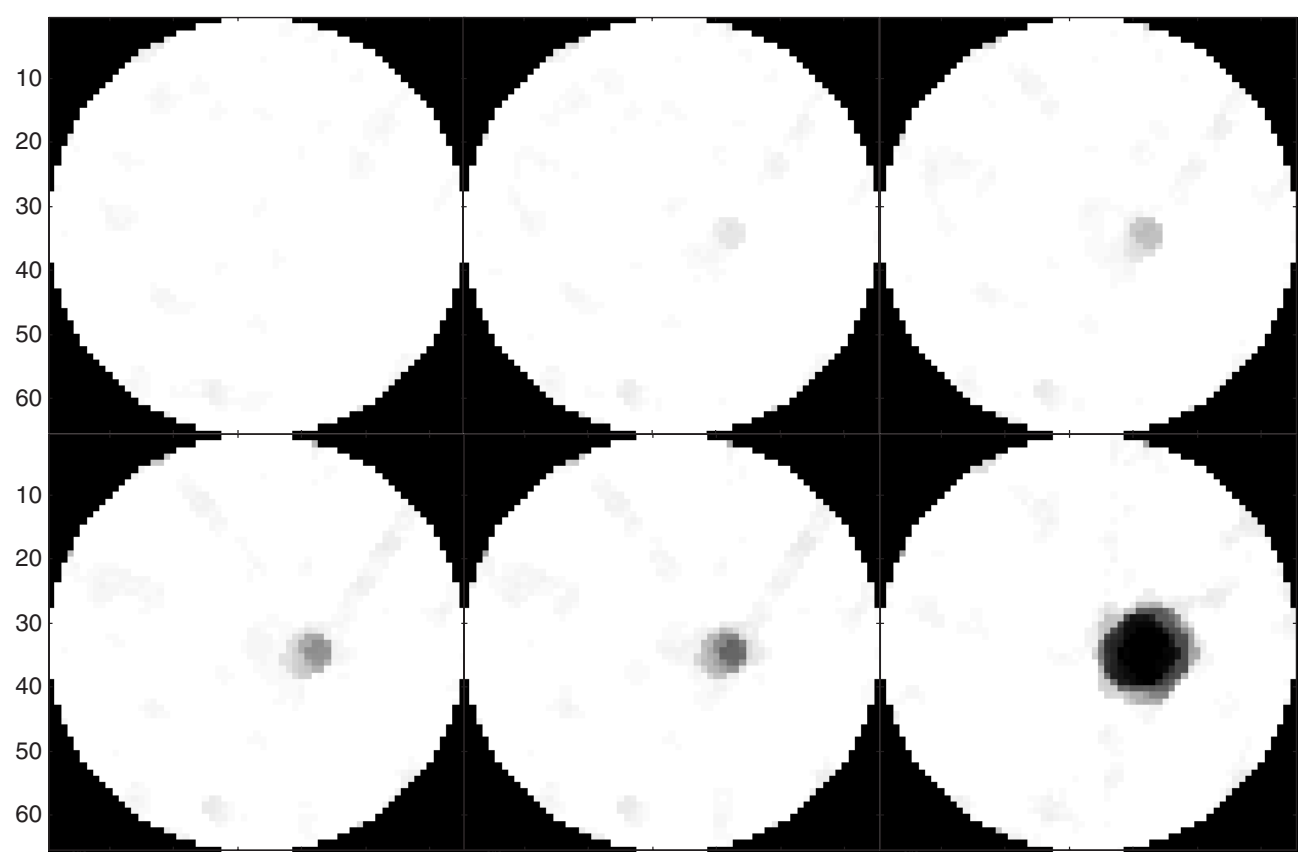

Figure 17. Reconstructed images of egg moving into the measuring plane (egg velocity $60 \mathrm{~cm} \mathrm{~s}^{-1}$ ). The top left image is the last image before the egg moves into the measuring plane. In the top middle the first sign of the egg is picked up. The following images show that the egg is moving upwards (time between frames: $5 \mathrm{~ms}$ ). The right-bottom image shows one of the last stages: the full egg diameter is in the field of view. The time difference between the last and last but one image is $170 \mathrm{~ms}$.

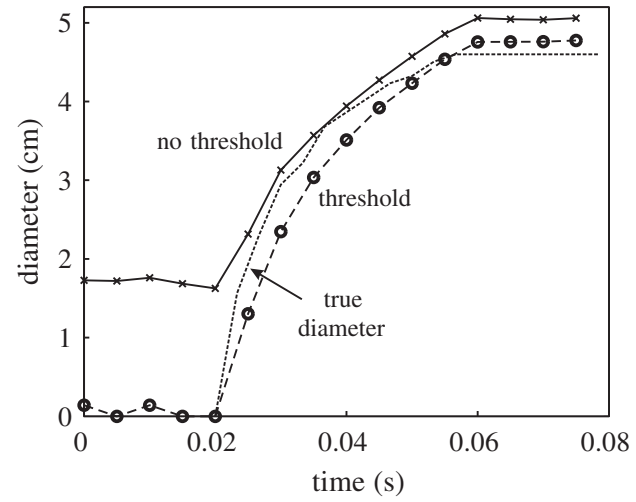

Figure 18. Diameter of the part of the egg in the field of view of the detectors $\left(60 \mathrm{~cm} \mathrm{~s}^{-1}\right)$. Only the first part of the motion, i.e. with the egg moving into the measuring plane is reconstructed. The crosses $(\times)$ indicate the diameter estimated from the data without applying a threshold value to the pixels; the circles do the same with a threshold; the dotted line is the diameter as measured with a marking gauge.

with the summation running over all pixels in the bed. Obviously, thresholding needs to be used, as otherwise noise everywhere in the reconstruction plane will bias the calculated diameter toward higher values. Typically, the above procedure without thresholding results in a diameter of $1-2 \mathrm{~cm}$ even if the egg is not in the reconstruction plane. The threshold levels have been adjusted manually, such that half of the reconstructed diameters before the egg has moved into the measuring plane are equal to zero. The diameter from the tomographic reconstruction is compared to the true diameter in figure 18.
Table 9. Egg diameter.

\begin{tabular}{lll}
\hline $\begin{array}{l}\text { Velocity } \\
\left(\mathrm{cm} \mathrm{s}^{-1}\right)\end{array}$ & Threshold & $\begin{array}{l}\text { Maximum } \\
\text { diameter }(\mathrm{mm})\end{array}$ \\
\hline 10 & 0.04 & 4.73 \\
20 & 0.11 & 4.73 \\
40 & 0.16 & 4.72 \\
60 & 0.12 & 4.76 \\
\hline
\end{tabular}

As can be seen from this figure, we slightly underpredict the diameter when the egg moves into the measuring plane and we overpredict it when the full diameter is in the plane. This is the general trend for most of the experiments, i.e. at velocities ranging from $10 \mathrm{~cm} \mathrm{~s}^{-1}$ to $60 \mathrm{~cm} \mathrm{~s}^{-1}$. At the moment the reason why is still unclear. The underprediction might have to do with the finite thickness of the measuring plane and the nonlinear response of $\mathrm{x}$-ray imaging. This point needs further attention. The other velocities result in similar graphs with the same order of magnitude for over and underprediction.

In table 9, the results of experiments at various velocities are collected.

As can be seen from the table, the egg diameter measured is independent of the egg velocity. We overpredict the egg diameter by $1-1.5 \mathrm{~mm}$. The underprediction in figure 18 is of the same order of magnitude.

\section{Concluding remarks}

In this paper, we have discussed the possibilities of a timeresolving X-ray-based tomographic scanner. Here, the key parameters are spatial resolution and measurement speed. We 
have used a standard medical $\mathrm{x}$-ray tube to produce an $\mathrm{x}$ ray fan beam. In combination with $30 \mathrm{CdWO}_{4}$ scintillator crystals, this forms our hardware. By using five different views (i.e. rotating the object under study over $72^{\circ}$ ) we generated enough views for a reconstruction. In this way, we mimic a scanner with five sources that simultaneously perform the measurements. We have found that we can reach a spatial resolution below $5 \mathrm{~mm}$ for a 23 column filled with polystyrene particles. Moreover, the measuring time could be kept below $5 \mathrm{~ms}$. This shows that $\mathrm{x}$-ray tomography can reach frame rates of 200 frames $s^{-1}$. It is expected that by reducing the amplitude of the voltage wiggle of the x-ray source a faster tomographic system is possible, with frame rates of the order of 1000 frames $\mathrm{s}^{-1}$. Alternatively, an extra detector, measuring the radiation from an x-ray beam that passes by the column, could monitor the actual source strength. This way the fluctuations could be corrected for.

The polyenergetic nature of the X-ray photons does not permit a straightforward use of the Lambert-Beer law in the tomographic reconstruction. However, a simple calibration of the response of the detectors was sufficient to overcome this problem. For an actual five-source system, the effects of scattering from $\mathrm{x}$-rays of one source on to the detector array of another source will increase the noise levels. This effect has to be investigated in future research.

From our experiments on voids we found that objects with a diameter of $5 \mathrm{~cm}$ could very easily be found in a $23 \mathrm{~cm}$ bed. Objects with a diameter of $2.2 \mathrm{~cm}$ were also detected, but their size is under predicted. Nevertheless, from our experiments we conclude that, in principle, an x-ray-based tomographic system with 5 sources and 30 detectors each is able to capture objects with a size of one-tenth of the fluidized bed moving through a fluidized bed with a diameter of a few decimeters.

Moving objects can also be reconstructed with good accuracy. We measured the passage of an egg shaped hollow object, with a maximum diameter of $4.6 \mathrm{~cm}$, at a vertical velocity ranging from 10 to $60 \mathrm{~cm} \mathrm{~s}^{-1}$. From the reconstructed images we could clearly see the passage of the egg. We found that the measured diameter is about $3 \%$ too high, regardless of the egg velocity. When the egg moves into the measuring plane, we underpredict the diameter. These experiments show that the system is fast and accurate enough to study moving objects.

\section{References}

Alenius S and Ruotsalainen U 1997 Bayesian image reconstruction for emission tomography based on median root prior Eur. $J$. Nucl. Med. 24 258-65

Alles J A and Mudde R F 2007 Beam hardening: analytical considerations of the effective attenuation coefficient of $\mathrm{x}$-ray tomography Med. Phys. 34 2882-9

Andersen A H and Kak A C 1984 Simultaneous algebraic reconstruction technique (SART): a superior implementation of the art algorithm Ultrason. Imaging 6 81-94
Beck M S, Dyakowski T and Williams R A 1998 Process tomography - the state of the art Trans. Inst. Meas. Control $20163-77$

Bieberle M, Fischer F, Schleicher E, Hampel U, Koch D, Aktay K, Menz H-J and Mayer H-G 2007 Ultrafast limited-angle-type x-ray tomography Appl. Phys. Lett. 91 123516/1-3

Bieberle M and Hampel U 2006 Evaluation of a limited angle scanned electron beam x-ray CT approach for two-phase pipe flows Meas. Sci. Technol. 17 2057-65

Bissessur Y and Peyton A 2005 A parametric reconstruction technique for planar electromagnetic inductance tomography Proc. 4th World Congress on Ind. Proc. Tomography (Aizu, Japan) pp 552-7

Brooks R A and DiChiro G 1976 Principles of computer assisted tomography (CAT) in radiographic and radioscopic imaging Phys. Med. Biol. 21 689-732

Duduković M P 2000 Opaque multiphase reactor: experimentation, modeling and troubleshooting Oil Gas Sci. Technol.-Rev. IFP 55135

Gordon R, Bender R and Herman G T 1970 Algebraic reconstruction techniques (ART) for three-dimensional electron microscopy and x-ray photography J. Theor. Biol. 29 471-81

Green P J 1990 Bayesian reconstruction from emission tomography data using a modified EM algorithm IEEE Trans. Med. Imaging 9 84-93

Herman G T 1980 Image Reconstruction from Projections-The Fundamentals of Computerized Tomography (New York: Academic)

Herman G T and Lent A 1976 Iterative reconstruction algorithms Comput. Biol. Med. 6 273-94

Jiang M and Wang G 2003 Convergence of the simultaneous algebraic reconstruction technique (SART) IEEE Trans. Image Proc. 12 957-61

Kak M and Slaney M 1988 Principles of Computerized Tomographic Imaging (New York: IEEE Press)

Kühn F T, Schouten J C, Mudde R F, Van den Bleek C M and Scarlett B 1996 Analysis of chaos in fluidization using electrical capacitance tomography Meas. Sci. Technol. 7361

Kumar S B and Duduković M P 1997 Computer assisted gamma and $\mathrm{x}$-ray tomography: applications to multiphase flow systems Non-Invasive Monitoring of Multiphase Flows ed J Chaouki, F Larachi and M P Duduković (Amsterdam: Elsevier) chapter 2 pp 43-103

Kumar S, Moslemian D and Duduković M 1995 A gamma ray tomographic scanner for imaging void fraction distribution in bubble columns Flow. Meas. Instrum. 661

Kumar S, Moslemian D and Duduković M 1997 Gas holdup measurements in bubble columns using computed tomography AIChE J. 431414

Mudde R F, Bruneau P R P and Van der Hagen T H J J 2005 Time-resolved gamma-densitometry imaging within fluidized beds Ind. Eng. Chem. Res. 44 6181-7

Mudde R F, Harteveld W K, Van den Akker H E A, Van der Hagen T H J J and Van Dam H 1999 Gamma radiation densitometry for studying the dynamics of fluidized beds Chem. Eng. Sci. $\mathbf{5 4} 2047$

Reinecke N and Mewes D 1996 Recent developments and industrial research applications of capacitance tomography Meas. Sci. Technol. 7 233-46

Shollenberger K A, Torczynski J R, Adkins D R, O’Hern T J and Jackson N B 1997 Gamma-densitometry tomography of gas holdup spatial distribution in industrial-scale bubble columns Chem. Eng. Sci. 522037

West R M, Jia X and Williams R A 2000 Parametric modelling in industrial process tomography Chem. Eng. J. 77 31-6 